\section{AN ENRICHING EXPERIENCE}

Accurate detection and quantification of low-abundance proteins by immunoblotting can be difficult since the amount of sample that can be loaded onto SDS-polyacrylamide gels is limited. However, methods to enrich for specific classes of proteins prior to gel electrophoresis can help overcome this problem. In this issue of BioTechniques, Azamat Karginov and Michael Agaphonov at the Russian Academy of Sciences describe a simple enrichment technique that greatly improves the immunoblotting of low-abundance yeast membrane proteins and is also superior to the standard method of boiling yeast cell lysates in Laemmli SDS gel-electrophoresis sample buffer. In the new approach, yeast cells are lysed in a buffer containing ammonium acetate, which protects proteins from degradation and facilitates membrane precipitation. The membrane fraction is obtained by a 10-minute centrifugation in a benchtop microcentrifuge and then resuspended in an alternative sample buffer containing SDS and a high concentration of urea, eliminating the boiling step that can cause protein degradation.

See "A simple enrichment procedure improves detection of membrane proteins by immunoblotting" on page 260 .

\section{DOUBLE-FISH FOR FROG EMBRYOS}

In situ hybridization (ISH) for the simultaneous detection of two different transcripts in Xenopus embryos has been tricky to carry out using conventional chromogenic substrates of alkaline phosphatase (AP) since the darker signal of substrates such as nitro blue tetrazolium/5-bromo-4-chloro-3-indolyl-phosphate (NBT/ $\mathrm{BCIP)}$ obscures the lighter signal from other substrates such as Vector Red. In addition, the opaque yolk platelets in the endodermal cells of Xenopus embryos interfere with signal detection. In this month's issue, Zachary Agricola and Sang-Wook Cha at Cincinnati Children's Hospital Medical Center describe their protocol for sequential double-fluorescence ISH (FISH) in Xenopus that resolves both of these issues. As substrates for AP development, they used Vector Red and NBT/BCIP, but these were visualized fluorescently by excitation with red and far-red light, respectively, which allowed the signal from each substrate to be detected without interference from the other. Coupled with treatment of the embryos by a clearing agent to render them transparent, this new approach enabled single-cell resolution of the expression of each target transcript.

\section{See "Two-color fluorescence in situ hybridization using chromogenic substrates in Xenopus" on page 263.}

Selected and written by Patrick C.H. Lo, Ph.D.

\section{DECIPHERING CYTOSINE METHYLATION}

The epigenetic modifications methylcytosine $(\mathrm{mC})$ and hydroxymethylcytosine $(\mathrm{hmC})$ play important roles in modulating gene expression. Although techniques have emerged to identify cytosine methylation patterns, they are limited in resolution, which makes distinguishing these marks experimentally challenging for researchers. Now, Kawasaki et al. present a method to simultaneously identify cytosine, $\mathrm{mC}$ and $\mathrm{hmC}$ at single-base resolution. Their approach relies on the enzyme DNMT1, which methylates the unmethylated cytosine in a hemi-methylated CpG but not in a hemi-hydroxymethylated CpG. Using DNMT1 alongside standard bisulfite treatment, the authors were able to identify $\mathrm{mC}, \mathrm{hmC}$, and cytosine sites in single assay. They validated the new EnIGMA (Enzyme-assisted Identification of Genome Modification Assay) technique by assaying a 200-bp sequence containing multiple cytosine modifications. This new approach should help researchers better catalog cytosine modifications and their effects on gene expression patterns.

Kawasaki et al. 2016. "A novel method for the simultaneous identification of methylcytosine and hydroxymethylcytosine at a single base resolution." Nucleic Acid Research. doi: 10.1093/nar/gkw994

\section{SINGLE-CELL, SINGLE-TUBE TRANSCRIPTOMICS AND PROTEOMICS}

A fundamental mystery of molecular biology is the relationship between gene expression and protein production. Attempts to understand how RNA and protein levels co-vary within cells have led to the development of new techniques for assaying these molecules simultaneously. Genshaft et al. now offer a method for profiling single-cell transcriptomes and proteomes in the same reaction. The key to their approach is the use of the reverse transcriptase activity of DNA polymerase for both complementary DNA synthesis and proximity extension assays. Proximity extension uses two antibodies attached to short DNA sequences. When the antibodies are close, the DNA sequences bind together and are extended by DNA polymerase to generate a protein-indexed DNA readout, which can be detected by sequencing. Using this method, the authors were able to profile changes in the transcriptome and proteome of a human breast cancer cell line in response to different chemical perturbations. This multiplexed profiling technique provides a new option for scientists exploring gene-protein expression relationships.

Genshaft et al. 2016. "Multiplexed, targeted profiling of singlecell proteomes and transcriptomes in a single reaction." Genome Biology. 17:188. doi: 10.1186/s13059-016-1045-6

Selected and written by Nathan S. Blow, Ph.D.

BioTechniques 61:223 (November 2016) doi 10.2144/000114469 